largest and most complete collection in existence of the results of gunshot injuries and of the diseases of armies. It is rich in illustrations of craniology and comparative osteology; and its specimens cover the whole field of medicine and surgery. Here is also the $\mathrm{Na}$ tional Medical Library, the largest exclusively medical library in the world, and, as a practical working library, the most valuable, owing to its immense collection of periodical literature, and the manner in which it has been indexed and catalogued. All praise and honor to the medical officers connected with the office of the Surgeon-General, to whom our profession and the country are indebted for the labor and skill that have made our National Medical Library and the Army Museum institutions of which we may justly be proud! Let us hope that Congress will not much longer delay in providing a commodious fire-proof building for the reception of the valuable collections which it would be impossible to replace, and which are of the greatest importance to the progress of medical science and education in this country.

The Naval Museum of Hygiene, although of recent growth, will be found to possess an interesting collection illustrating sanitary science, together with a good library. The National Museum and the Museum of the Department of Agriculture contain also much that is of interest to members of our profession.

To my congratulations are to be added my thanks for the great honor conferred on me by my election to the Presidency of the Association. My grateful appreciation of the honor is enhanced by the fact that I did not seek nor desire the office. I should have been glad had the selection fallen on one better prepared to perform the duties of a presiding officer. I can only say that, relying on your indulgence and forbearance, I shall discharge these duties to the best of my ability.

May this thirty-fifth annual meeting of the Association be characterized by harmony and good feeling! With the blessing of Providence, may its proceedings strengthen the attachment of the American medical profession to the Association, extend the boundaries of our knowledge, and promote the welfare of mankind!

\section{ADDRESS ON PRACTICAL MEDICINE.}

BY JOHN V. SHOEMAKER, M.D., CHAIRMAN OF THE SECTION ON PRACTICAL MEDICINE AND MATERIA MEDICA, MAY, I 884 .

Gentlemen of the American Medical Association:

A year ago you conferred upon me the honorable position of Chairman of the Section of the Practice of Medicine, Materia Medica, and Physiology of your Society. As Chairman, it is made imperative upon me, by our By-laws, to lay before you a résumé of the progress made and the new discoveries an- nounced in these branches of our science during the past year. The responsibility of this duty, in view of the numerous investigations and important discoveries by the members of the medical profession throughout the civilized world, and the steadily increasing volume of medical literature, etc., renders the task more and more difficult with each succeeding year; for as new theories arise and supplant older ones, they, in turn, will be superseded by others yet unheralded. The unbiased gleaning of what has been done is a feat of no mean proportion, and if I should not do full justice in my criticism of any particular one, or in the mention of others, I beg your indulgence in advance.

Medicine, at the present time, has a tendency to elevate itself beyond the narrow sphere occupied by it years ago. Empiricism is no longer recognized. The ultimate investigation of the cause of disease, and the remedial measures suited to it, seem the absorbing object. While our present scientific system of medicine has many followers among the sectarians, sprung from its maternal lap, it still stands proudly alone, brighter and fairer in its record than ever before. The scepticism which has arisen from heresy has had a most salutary and purifying influence on it, and has led us, again and again, to review the course we are pursuing, in order to be assured that there is no deviation from the right direction.

Sceptics hold that with all our efforts, no advance has been made upon the inroads of disease, and that mankind is as much subject to its influence as when medicine blindly groped its way. This allegation I most certainly deny. No longer are whole continents devastated by disease; no longer are epidemics the terror of nations, nor countries made uninhabitable to the human race; for with an intimate knowledge of disease and its origin, scientific medicine has adapted measures to its stay, its relief, and its extermination. And all this has been done notwithstanding population is steadily increasing, and with it, also, the danger of contamination from disease-breeding germs, multiplying at an unduly increasing ratio; that the desire for social intercourse makes itself manifest by closer congregation in localities illy suited for hygienic protection; and with it the stationary, if not reduced rate of certain classes of complaints, must certainly be regarded as a veritable triumph for medicine, and the necessity for continual effort for its advancement.

The means for making medicine more thoroughly understood and serviceable are, without doubt, the general distribution of its principles and conditions by the wide development of medical literature. A great source of satisfaction is the high standard which it has attained with its wide diffusion, thus enabling the physician everywhere to be in close intellectual communion with his fellow-workers. The time when medical knowledge was kept a family or a trade secret, was less productive of true medical advance than any other period of man's existence. With the universal spread of knowledge alone has medicine risen from empiricism, until to-day it may be called a scientific system indeed. It is a matter of congratulation that this great, and the most prominent, 
association of practitioners of inedicine of our country has launched a journal, which has taken the place of the time-worn Transactions, in which the valuable investigations of the members of our Association were quietly buried. Not alone has our science been advanced by the improved and increased periodical medical literature, but numerous publications on special subjects and branches have appeared during the past year, which have been largely instrumental in promulgating and advancing new theories.

It is the studious investigator, lrowever, who, with untiring zeal and devotion, has disinterestedly employed his time in the study of health, disease, and its curative measures, that medicine has to thank for its principal advancement during the past year. The student in the laboratory, at the microscope, as well as in the actual field of devastating epidemics, is the benefactor of whom too much cannot be said in praise. While it is not our province to enumerate or name them here, it becomes certainly our duty to mention that noble hero. Dr. Thullier, of Paris, who, with the French Cholera Commission, penetrated into the very pest-houses of Egypt to serve the cause of medicine, and gave up his life there, a victim for our enlightenment. His memory should be a lasting one with physicians all over the world as a substantial monument in connection with many other great heroes in our ranks.

\section{PHYSIOLOGY AND PATHOLOGY.}

I can say but little of the progress of physiology during the past year. The field is one of vast expanse, and the observations that have been recently put forth have not, as yet, stood the test of time, so necessary in this branch of our science.

In the field of pathology and pathological research the study of the infinitesimal has attracted great attention. Humeral pathology of old has had its day. Anatomico-pathology now seems fast to lose its hold upon our workers, in view of the discovery of the constant appearance of micro-organisms as concomitants of special types of disease. Grosser lesions appear to have lost their interest and importance in comparison with the invading hosts of parasitic enemies to the organic structures. Though their presence was known for years, as has been demonstrated by older observers, we are riding now on the very crest of the billow of excitement that they are creating in medicine. Tempora mutantur! Will it be rash to predict a downfall of the omnipotent rule of these minute Vandals who overawe us with their menaces? That they exist there is no longer any reasonable doubt. 'They have been demonstrated, classified, propagated, and shown to be constant with certain features of tissue changes. Their causative influence is as yet a matter of grave doubt, and one that in accordance with close observation will probably not be soon cleared up. If all that their enthusiastic discoverers and experimenters claim proves true, medicine will resolve itself into a system of micro-botany, leading to the study of the character and habits of its subjects, and the antagonistic elements to their existence.

Of the subjects prominently brought to the attention of the profession and exciting universal interest, more than any other is that of the bacillus theory of tuberculosis and its contagious character. While the presence of micro-organisms in other diseases is being investigated and studied extensively, there is none which has as great a bearing and caused so much comment as this one. Only a few years ago Koch stood almost alone in its advocacy. Now there are numbers of unbiased observers who confirm his famous discovery. Foremost among these is Watson Cheyne, who visited Koch's laboratory for the express purpose of investigating the subject. His report fully confirms the facts set forth by the great investigator, besides demonstrating the constancy of the occurrence of bacillus in all tubercular structures, as well as the relative position of the parasitic organism to cell-structure. Similar confirmatory evidence has been produced by Pruden, Dreschfeld, West, Gibbs, Heron, Wipham, Meissen, and others, from a clinical view of the constant presence of the bacillus in the sputa of phthisis ; while Charnley Smith has even ingeniously demonstrated its presence in the expiratory air of consumptives by filtering it through gun-cotton, dissolving it in ether, and then fixing it on microscopical slides for inspection.

Dr. Austin Flint, Sr., accepts the new theory as well as the ætiological character of this micro-organism when added to predisposition, leaving the subject of contagion dependent upon the causative element combined with the favorable soil for its propagation, ascribing the usual absence of contagion to a lack of predisposition, and considers the presence of the bacillus as one of the most essential elements for the diagnosis of phthisis. Drs. Janeway, Welch, and Peabody, well-known authorities on this subject, concur with Dr. Flint in his views; likewise Dr. H. C. Ernst in his contribution presented to the Massachusetts Medical Society, besides numerous other investigators, both here and abroad.

The presence of the bacillus has been observed in other tubercular lesions than the lungs: as in the mouth and soft palate, by Guttmann and Finger; in the skin by Cornil ; in lupus by Demme; in the uterus by Lindsey Stephens; in the urinary tract by Rosenstein, Babés, and Cornil, as well as in rectal abscesses by Smith. Dr. Wilson Fox admits the fallacy of his former experiments, and accepts the theory of the contagious character and inoculability of the virus, and that injury to the rodents is not followed by tuberculosis, as previously expressed by him.

Now, while the adherents to Koch's theory have increased wonderfully, there are those not wanting who are open in their criticism, and opposed to the acceptance of anything like a bacterian origin of tuberculosis or other diseases. Their opposition is not based merely upon assertions or clinical evidence, but the result of years of observation and experiments conducted here, as well as at the very birthplace of the bacillus theory. Amongst those most prominently arrayed against it are Spina, of Vienna, Finkler, and Eichler, who pronounce the staining methods of Koch as fallacious and misleading, while Feltz admits his failure with cultivation by following Koch's method.

Formad, in this country, has made a most vigorous 
attack upon it, and has referred the ætiology of tuberculosis to histological changes rather than to parasitic invasion, in which deduction he is supported by many eminent pathologists, such as Longstreth and others. In his denial of the contagiousness of tuberculosis he is in accord with the opinions of Virchow, Recklinghausen, Stricker, Gull, Williams, Watson, Paget, Humphrey, Richardson, Bennet, Hiram, Corson, Trail Green, N. S. Davis, H. F. Campbell, and others. Thus, it will be seen that this subject is by no means disposed of yet; and it will undoubtedly form one of the main and interesting points of our discussions at this meeting, in which some of our most prominent pathologists, practitioners and teachers have promised to take part.

The bacillus tuberculosis theory, however, is not the only one that has excited attention. The bacterial origin of other diseases has been earnestly advocated as well. Thus, a micro-organism has been found and studied by Friedländer as a micrococcus of pneumonia, possessing distinct capsules under certain conditions and at certain stages, which he verified by cultivating processes and inhalation of impregnated air, and was enabled in that way, in certain animals, to produce red infiltration of the lungs, and evolved reddish infiltration of the pleura. The capsule was easily seen by means of staining fluids, but does not exist in the cultivated micrococcus, but seems to be reproduced in the experiments upon animals, and appears to be mucin. Though reported before by Giles in the blood of pneumonic patients, where Friedländer failed to see them, they were conclusively demonstrated by cultivation and inoculation to exist. This may serve to explain the occasional epidemic form of pneumonia in certain localities. Buchanan and Russell have described cases of infective pleurisy in which micro-organisms appeared in the effusion. A bacilIus of syphilis has been described by Morison, of Baltimore, as occurring in chancres, condylomata, etc., but apparently different from that described by Birch-Hirschfeld; while the micrococcus of gonorrhœa has been cultivated by Burckhart, as well as Sternberg, and successfully inoculated. Again, micro-organisms have been found and claimed as the cause of pertussis, while cases of nephritis and purpura have been attributed to the same cause, and even the vesirles of impetigo contagiosa and herpes labialis, it is asserted, contain them. A micro-organism is said to have been detected in the blood of patients ill with typhus; and Marigliano appears to confirm Klebs and Eberth as to their presence in the blood of the spleen of typhoid-fever patients. The bacillus of leprosy has again been reported by Hausen and Thin, as well as that of scarlet fever by Crooke. The discovery of the bacillus of glanders by Bonchance, Schutz, and Loeffler has been confirmed by successful inoculation. Erysipelas has been demonstrated by Fehleisen to be due to the growth of micrococci in the lymphatics of the skin, and by inoculation and cultivation he successfully introduced that affection both in animals and man. The work of Pasteur and Koch has been directed with success toward the study of the attenuation of virus; and the latter's labors, as head of the German Cholera Commission sent to
Egypt and India, have been crowned by the discovery of the specific micro-organism of cholera. The constant presence of the cholera bacillus in the intestines and ejecta, seems to leave no doubt as to the important part they take in that scourge. While Prior has found a micrococcus in the coats of the intestines and stools of dysentery, and numerous organisms have been for years declared to be the cause of cholera, it is due to Koch, for his special skill in this field of research, to accord him the honor of the detection of the special micro-organism, to which may be fairly attributed the pathological changes in these structures.

That the origin of yellow fever should arise from this same cause is readily obvious, when we consider its infectious nature. Though this has been claimed to be the case by Carmona del Valle, and called by him " peronosperma lutea," there seems to be little tangible evidence for it aside from the experiments of Domingos Freire, of Rio de Janeiro, who showed, experimentally, that blood of dead yellow fever patients, inoculated into animals, caused rapid death ; and that their blood had, in turn, similar effects. $\mathrm{He}$ attributes this to the presence of a cryptococcus, for which he proposes the name of cryptococcus xanthogenicus; and which he also claimed to have found in the earth of infected districts. While these demonstrations prove scarcely anything without proper cultivation and successful inoculation, they point, nevertheless, to a fruitful field for research.

\section{MEDICINE.}

Medicine, in a word, comprises everything relating to the healing art; necessarily we will have to give it here a closer meaning in its relation to clinical observation only. The pathological conditions, so nearly allied to it, we have treated of before. The treatment of disease we shall speak of hereafter. Hence, it remains but to call your attention to new phases of disease, of their diagnostic characteristics, and their prognostic chances.

The knowledge of nervous affections, though it has not progressed with the giant strides of other branches. of disease, is steadily advancing and emerging from its mysterious enfoldings. The lucalization of cerebral functions, though now generally accepted, has advanced us practically but little, though the trephine has daringly opened to the neurologist the hidden regions of dura mater and gray matter. Locomotor ataxy has presented in its chronic state three diagnostic points for distinction: that of Duchenne, with the characteristic symptoms depending on the lesions of the columns of Burdach ; another depending on diffuse sclerosis of the chord and medulla oblongata, although principally limited to the posterior columns of the former, differing from it by its first occurrence during puberty - sensibility not being affected until late in the disease - and that the uncertain gait and step is not increased by shutting the eyes. A third subdivision is made of the ataxy of the periphery, described by Dejerine as an atrophy of the peripheral sensory nerves.

While the treatment of the insane has so vastly improved, it is to be regretted that our knowledge of 
the rtiological factors of psychical disease does not keep pace with it, and from this cause, no doubt, arises the difficulty experienced in the diagnosis of such cases, which give rise now and then to conflicting opinions in law courts, and which are interpreted to the great disadvantage of medical science. The interesting experiments of Brown-Sequard on reflex irritation, and the production by it of profound anæsthesia, bordering on catalepsy, should throw some light on the unravelled secret of hysteria and its allied affections.

The investigations and researches in the bacterial origin of phthisis would of necessity call for a reorganization of its clinical aspects. It has attracted the attention of eminent scientists, both pro and con. The bacterial development from extraneous sources would constitute it as contagious per se, and be in accord with the theories of its advocates; while those opposed to it have most ingeniously brought forward arguments, which, if substantiated, would upset our principles of organic law. The origination de novo of the bacillus, as claimed by Bouchardt, would depend on metamorphoses, no longer admitted, and certainly not understood nor explained. Guerin, in supporting his doctrine, admits its transmissibility by classifying the tubercular structures as one non-transmissible and another transmissible; although the latter he holds communicable only through putrid infection. After all, these arguments fail to meet the issue, which, in view of the wide spread of this disease, is of paramount importance, in so far as it forms not alone the indication for antiseptic treatment, but the development and propagation of the disease itself.

Typhoid fever has arrested the attention of the profession to some extent, with a view of learning the real danger hidden in it, which is now almost universally conceded to be the accompanying hyperpyrexia, giving rise to the tissue changes. Excretion of nitrogenized material, and non-assimilation of the modification of these under the antipyretic treatment can be accepted as a guide to this conclusion. The treatment by cold baths, of all antipyretics, is now thought to be the most serviceable, if we can trust to the statistics of recent investigators, as well as the thorough researches of Sassetzky in the analyses of the excreta. If, as is stated, the mortality caused by enteric fever in London was ordinarily from $I_{5}$ to 18 per cent. of the population, and that it has now been reduced by means of the cold water treatment to about io per cent., it must certainly be regarded as a great improvement.

Of renal diseases, albuminuria has long been the subject of scientific inquiry, being considered more and more of nerve origin. Its close relation to diabetes, with mutual interchange in certain affections, has brought forward the proposition that both albuminuria and glycosuria may be produced by irritation of certain parts of the floor of the fourth ventricle. And the proposition is well established.

The hypertrophy of the muscular coat of the arterioles, and the increased tension of the vascular system, are also claimed to be due to irritation of the vaso-motor centers of the medulla. The latter especially has proved valuable in modifying the treatment of albuminuria. The neural influences seem primarily, and the renal disorganization from distension of the glomerular vessels progressive steps therefrom. That the toxic influence of certain substances, as well as severe febrile affections, is productive of albuminuria is well known, and a recent instance has been cited where it occurred after varicella. Peptonuria has been investigated and found to co-exist with suppuration from various causes, or with large exudations. Jaksch claims that it is due to the reabsorption of inflammatory products rather than to any pathological condition of the kidneys. The examination of urine for the purpose of detecting pathological products has probably further advanced during the past year than ever before. The old and common tests by nitric acid and heat are said to lack delicacy, and do not indicate albumen unless present in large quantities. The acidulated brine test, the tests with picric acid, Tanret's or potassio-mercuric, the sodium tungstate, the potassium ferrocyanide tests, in their solid or liquid form, or spread on paper for convenience sake, have no doubt their advantages, especially in point of delicacy, so that they seem an improvement on the older methods; but this very delicacy will, at times, mislead the practitioner, who, by the presence of peptones, mucin, urates, and medicinal bodies may form a hasty and erroneous diagnosis; besides, albumen might be detected as a normal constituent of the urine, when the older methods properly applied would serve as an indication of pathological change only. A question of greater importance for the medical chemist to solve is rather as to the quantity of albumen present, than to discover its probable incidental existence. The same may well be said of the new tests for sugar, such as diazobenzol-sulphuric acid, citric acid and indigo, only that they do not mislead, but serve well for qualitative tests at the bedside. For quantitative tests there will probably few outrival the older methods, or the easily applied and accurate polariscope.

The subject of myxœdema has received new impetus with our confréres abroad, both through the pathological researches of Henrot and of Kocher, in connection with the extirpation of the thyroid gland in a number of patients who subsequently showed a cretinoid condition, and the confirmation of an atrophied state of this gland in subjects of this disease. If the committee of the London Clinical Society, having this matter in charge, will solve some of the points concerned, it will shed much interesting light on its functions.

The disease known as actinomycosis, and described by Ponfick as peculiar to horses and cattle, has been exhibited by Treves in a man, whom he brought before the London Pathological Society. All stages of the disease, from minute solid growths to large suppurating and sloughing masses, were present.

The grave accusation cast against American pork by some of the European authorities makes it necessary that I should say a few words here upon the subject. Trichinosis is with us a very rare disease indeed, in comparison to its frequent occurrence in 
Continental Europe. In spite of the prohibitive edict against our pork in Germany, quite an endemic of trichinosis has prevailed there of late. As there have been isolated cases reported with us, I will therefore refrain from any attempt at defence, but simply remark that the mortality from trichinosis in this country, in comparison with Germany, would lead us most certainly to discriminate in favor of our product to that of the foreign-even if we do not attribute our comparative immunity to the more civilized manner in which we partake of it than the semi-barbarous fashion of eating it uncooked. French physicians, in the Academy of Medicine, of Paris, have already given their opinion that American pork is not as dangerous as the trichiniphobist would have us believe, and recommend that the prohibition to its importation be removed.

The epidemics of small-pox, which for some years have swept over this country, and have visited almost every town and hamlet, have considerably abated, but few cases, comparatively, being reported now. The questions, if the disease has exhausted the material for its propagation, or if the vigorous vaccination insisted upon by the medical profession, has successfully stayed its ravages, are points which are not yet settled. Again the subject of a common origin of vaccinia and variola has been brought forward, and Voigt states that variola is inoculable into cows, and if used for protective inoculation with man it is equivalent, and even preferatle, to vaccine, provided that the virus be far enough remote from its first source, else variola, with all its characteristics, may be developed.

In regard to scarlatina much interest has been attached to its prevalence among animals - as the horse, cat and dog. It has been claimed that contagion was carried both from them to man, and vice versa. The pinkeye of horses has been stated to be in many cases nothing else than equine scarlatina, readily contagious, while cases are cited every now and then where the source of the disease unmistakably pointed to the domestic feline or canine pet. Of the greatest interest herewith are the experiments of Stickler with the inoculation of the nasal mucus of horses, suffering from scarlatina, into rabbits and dogs, which produced a modified scarlatiniform exanthem, accompanied by fever and some enlargement of the glands. Blood, from a case of confluent scarlatina, afterwards inoculated in those animals, failed to bring any results. Stickler repeated the same experiments on twelve children, but failed to produce scarlatina in any of them by inoculation with scarlatinal blood, and arrived at the conclusion that the subcutaneous injection of scarlatina virus from the horse is not followed by harmful results, but that it produces a circumscribed eruption like mild scarlet fever, and that such inoculation confers an immunity from scarlatina.

The epidemic which excited the most alarm in its disastrous results, and caused the greatest anxiety as to its spread, was the late outbreak of cholera in Egypt. That country is proverbial for its filth, and, as a consequence, its prolific breeding of epidemic diseases. Although cholera has periodically visited both this country and Europe, its terrible fatality has never been felt so severely as in Egypt, which has suffered from its late outbreak there. 'The lack of proper drainage, and the use of water contaminated with offal and excrementitious material, are well calculated to give impetus to this disease. From the reports of Sir William Hunter and other gentlemen of the European Sanitary Commissions, we learn of the most loose and incomplete hospital arrangements, while the harshness of its quarantine enforcements arises more from individual egotism and fear than the desire to stamp out the germs of the disease, which are hatched by the stifling heat, from the filth and offal exposed everywhere to view, and offending the nostrils at every step.

Yellow fever, as usual, made its appearance in some of the Southern ports, and while it showed a tendency to a malignant character, and indeed produced great ravages in the Pensacola Navy Yard, it is due to wise quarantine laws, and to the unflinching energy of the surgeons in charge of our naval and marine hospitals, that the disease was not represented in a palliative light, and thus allowed to spread beyond its original cordon. Much praise is due to Dr. Guiteras, of the Navy, for his moral courage in facing the issue in its true light, and thus sparing the South the disastrous experiences of former years. The attempts to fight yellow fever by calling it by another name are fraught with disastrous consequences, and should under no consideration be countenanced. That it is both infectious and contagious can no longer be denied, and, if not accompanied by urinary suppression, it is a disease which otherwise shows a fair percentage of recoveries under proper treatment.

\section{THERAPEUTICS.}

It may be said of therapeutics, that while in past years they were ignored to a great extent by many physicians and scientists, they have undoubtedly now assumed new features entitling them to a prominent position. Promiscuous dosing and empirical drugging are no longer recognized, but the application of the physiological action of remedial substances to a pathological condition are the therapeutics of the present and the future.

Among the permanent remedies which have found approval during the past year, there is probably none of greater interest than what promises to be one of the most powerful and reliable antipyretics, a rival to, and perhaps surpassing in its effects as a febrifuge, quinine. It is called kairin. Although it has as yet found but little practical application in our own country, the reports concerning it from abroad are such as would make it desirable for us to give it a thorough, practical test. It may not be all its admirers claim for it, but enough has been learned to place it prominently in the class of antipyretics. It was discovered by Dr. O. Fischer, and described chemically as an oxethylquinoline hydrid hydrochloride, a coal-tar derivative. It was investigated by Filhane, Sassetzki, Knipping, Girat and Guttman, with satisfactory results, while Reigel reports adversely as to his success. Notwithstanding the favorable properties it undoubtedly possesses to reduce fever temperature, it should 
be exhibited with caution, on account of its cardiac depressant action. Although it promises a remedy of the greatest value, sufficient investigation as to its physiological effect seems to be still wanting.

Another very interesting remedial substance which has been presented to the medical profession as experimented with, is the paraldehyde, a substance isomeric with aldehyde. It is asserted by Dr. Langreuter that it possesses powerful hypnotic properties, with no abnormal phenomena excepting a slight and irregular pulse. 'The sleep produced by it is readily induced by doses of about six grams ( $\mathrm{I} / 2$ drachms), generally giving a full night's rest, and when sleep is not produced, still a quieting influence is exercised. It is claimod for it, that it produces no cardiac depressant effect, but from its chemical source this seems doubtful. Dr. J. C. Wilson's experiments in this respect corroborate the above views. It is also claimed for it that a lethal dose suspends the functions of the medilla, and the action of the heart ceases after respiration; likewise that it acts upon the cerebral hemispheres, inducing sleepy drowsiness without preliminary excitement. While cardiac depression from if has been observed, it is said that neither nausea, depression, headache, constipation, nor other unpleasant after effects are produced by its use. It has a controlling action over some branches of the fifth pair of nerves, and seems to correspond in this effect to one of its derivatives-butychloralhydrate, or the so-called croton-chloralhydrate. While no doubt a useful hypnotic, it seems scarcely destined to supplant chloral for that purpose. Its close identity with the latter, however, therapeutically, is its antagonism to strychnine, and vice versa, for which property it has been studied and observed by Professor Cervella, who demonstrated that the seat of the opposing action is the spinal cord, the reflex irritability of the medulla oblongata being increased by strychnine and decreased by paraldehyde.

By far the most useful, as well as the most interesting effect produced by any new therapeutic agent given to us during the past year, is the Abrus precatorius, the jequirity bean of Brazil. The remarkable virtues of this drug have not alone been due to the curative effect it has exerted when applied as a remedy for pannus and other eye affections, and for which it has been reported as being a most extraordinary curative agent, but principally to the fact that in it we have pressed into our service the ubiquitous microbe, which is receiving at the present time so much attention. Sattler has proved beyond a doubt, that the watery infusion of the substance is a culture fluid, a nutrient field for certain atmospheric germs, which derive therefrom their pullulation, confirming the theory of Pasteur of the agency of ærial microorganisms in bringing about fermentation and morbid changes. That this is not mere speculation was again confirmed by the experiments of Cornil and Berlioz, who produced, by hypodermic injections of the infusion of jequirity, inflammation with suppuration and gangrene of the skin in rabbits, frogs, etc. Those animals which survived were not affected by a second operation, which has led some to the conclusion that the first had acted similar to a vaccination. In ani- mals to which the infusion was given in only a fourth of a drop, injected into the pleural or peritoneal cavity, it caused inflammations, and the microbes were found throughout their circulation in immense quantities. A drop of their blood injected in turn in a frog set up septic infection, and caused the presence of numerous microbes, but if the bean were given as food no infection occurred.

A matter of interest in therapeutics of the past year was the investigations of DaCosta in regard to the salts of nickel, and especially its bromide. While the other salts proved of little, if any value, this bromide was found to possess the properties of the bromides generally, but in much smaller doses. It is claimed that it lowers the temperature, does not influence the pulse, nor affect the excretions; that it is a nerve sedative, without exercising a weakening or depressing influence, and of especial value in eprlepsy.

The bismuth salicylate is also a new arrival among us, which has been used in the treatment of various forms of diarrhœea and typhoid fever; the latter disease, it is asserted, it will abort under favorable circumstances, but this claim needs further confirmation.

Nitroglycerine has again come to the surface, and new and striking results, as well as a larger sphere for its action has been developed, along with the cioser study of its physiological action. The opinion of Robson in regard to its value in acute and chronic Bright's Disease has been confirmed by its action on the vascular apparatus. Its effect in albuminuria has been recently investigated by Bartholow, who states that it reduces the vascular tension more than any other remedy; lessens the work of the heart by removing the inhibition exercised by the pneumogastric nerve; and that by its action he has not alone seen remarkable instances of relief, but even permanent cures. In angina pectoris it seems second only to amyl nitrite; and while it does not arrest the paroxysm as promptly as the former, it possesses a curative action, and can be used to prevent future atacks. For that purpose it is given daily to restore the diminished vascular pressure. The results attained with this agent, and the study of it along with the nitrites as a class, and their effect upon vascular tension, has recently developed remarkable and interesting facts in regard to one of this class, the sodium nitrite. The dose of it had been stated as about twenty grains, given for a long period with impunity. It appears that this applied to an impure article; that the pure sodium nitrite is possessed of powerful and even toxic action, as reported by Ringer and Murrell, and the commencing dose should not exceed two grains. In connection with this subject it is proper to allude to the controversy arising from the publication of the experiments of the gentleman last named.

Dr. Murrell was severely attacked for his wanton experimentation, as it was termed, on hospital and charity patients, not alone by laymen, but also by members of his own profession. Just how these gentlemen expect to derive knowledge without " experimentation," is not obvious. Perhaps they would have preferred to have been the victims of an erro- 
neously stated dose than to have the truth developed by scientific research. Dr. Murrell is entitled to the thanks of the profession for his demonstrations and important deductions, rather than virulent abuse; for with a cessation of "experimentation" the progress of medicine must also cease.

Recent investigations in the treatment of albuminuria, by Bartholow, also show the great utility of the chloride of gold and sodium in this disease, both in the subacute and chronic form. He states that " the earlier it is given the better, if structural changes are to be prevented or arrested, and its curative action will necessarily depend on the extent of the damage already inflicted on the kidnevs."

It is well to mention, also, that among others, attention has been directed to the following remedies: Hyperosmic acid seems to have attracted notice, both for parenchymatous injections in tumors as well as for obstinate neuralgia. Convallaria majalis and its active principle, convallamarin, after a thorough test is, according to Robson, inferior to digitalis in its action. The digitalis group, though not definitely limited, seems to have been positively defined as contracting the blood-vessels by their effect on the involuntary muscular tissue of these organs. Hypodermic injections of pilocarpine in myxoedema are reported as being very useful. Barium chloride has been studied physiologically, and while its therapeutic value is undetermined, it is proved to possess a powerful action quite different from the calcium chloride, with which it has been classed, and its resemblance in its action to digitalis is confirmed. Croton oil has been found to consist of two therapeutically different principles, one of which represents a safe and useful laxative, while the other is stated to be a vesicant and intestinal irritant. The use of potassium permanganate, hypodermically injected as an antidote for the bite of the cobra and other venomous snakes, has again been brought forward, and though its efficacy has been doubted by some, by others it is asserted that if immediately or shortly. after being bitten it is injected into the area of the wound, it will neutralize the poison, while it will not counteract it when reabsorbed and constitutional symptoms have set in. In connection herewith, Sir Joseph Frayer, who has made an extensive research into the nature of snake poison, states in a recent communication before the Medical Society of London: "The result of my experience is that, so far, no physiological antidote to snake virus is known, and that when the full effect on the respiratory center is produced, remedies are of little, if any avail; albeit, when the poison has entered in small quantities, treatment may be of service on general principles."

The vast research in the field for micro-organisms as causative elements of disease and the discoveries made therein, have given a new impetus to antiseptic treatment in every branch and affection. Antiseptic inhalations for pulmonary disease have proved of value, whether the germ theory be sustained or not. Carbolic acid and creosote are serviceable in typhoid fever, and other antiseptic agents are daily employed with equally good success. If their favorable action prove antiparasitic or otherwise, cannot at this time be ascertained, but in view of the results further investigations are indicated. Amongst the new antiseptics, naphthaline and naphthol have been mentioned as of great service; in regard to the latter, $I$ have ascertained that if pure and properly prepared, it seems devoid of the toxic effects claimed for it.

A new feature of the greatest importance, which was first agitated by the British Medical Association, and brought before us at our last meeting, is that of collective investigation of disease. 'There is no denying the fact, that not alone have our statistics of disease, its ætiology, course, and termination, been very loosely collated and obtained from sources which altogether inadequately represent the condition of our country, as well as the domicils of the majority of its inhabitants, but that we have, as a consequence, no general idea as to the amount, the severity, and issue of disease, even if we take for granted that the reports of the various health officers are tolerably correct in regard to fatal results. The reports already received from our English brethren have demonstrated their value, and prompt us to a similar inode of investigation.

This system of collective investigation is also pursued in Germany, and it will, no doubt, ere long comprise all the countries which have an organized medical profession. The systematic collection of therapeutic results will alone be invaluable by showing statistically the worth of certain medicinal measures and their comparison in effects with others.

And now, in closing, I would remark that I have no new plans or propositions to offer for your consideration. The Section of Medicine continues to grow and attract more attention with each succeeding year. It is necessary for you, however, with this growth and development, to direct the attention of those entering the profession to the necessity of thoroughly mastering the details of all its different branches; above all that of general medicine, before entering into any specialty. There appears, just at this time, and it is for this reason that I refer to the subject, a desire on the part of a few physicians to gather in cliques, and, with shut doors against their fellow-practitioners, settle to their own satisfaction momentous questions in this or that special department which they affect. Such organizations are generally composed of a few medical authors and teachers and some of their friends, who think themselves immeasurably superior to the profession at large, and would create an aristocracy, as it were, in one of the most democratic of sciences and professions. Here lurks the danger to medicine as a science. When in centuries back the knowledge of special branches of medicine was kept a close secret, and transmitted as an heirloom in certain families, or hidden in the parchments of monastic libraries, the general practitioner was excluded from obtaining knowledge from these sources, and thus benefiting mankind generally, but was compelled to grope his way in darkness and trust to his own intellectual force. At that time it was thougnt impossible for the ordinary physician to handle the obstetrical forceps or cure favus-yet to-day both are a common practice. To that undesirable point shall we drift again if we will admit of this exclusiveness. The ten- 
dency of these cliques is to keep intact their sacred circle for the use of themselves and their friends, not to study and teach by their assemblies, but to force the general practitioner to call to his aid these special oracles in every possible case.

Medicine, to be beneficial to humanity, must be open to one and all, and if its practitioners are to be excluded from medical societies and their meetingsnot from a lack of knowledge and good standingsuch societies have outlived their usefulness. Special studies may be made by physicians in certain directions, but the advantages of their research and study must be verified by their practical demonstration through the general practitioner. Without a thorough knowledge specialism avails little. This powerful organization should represent every branch and speciality of medicine, and its knowledge should be open to all its members. We are all entitled to the benefits derived from associated investigations. Outside organizations on special subjects will detract from the interest and advantages of our meetings, and the younger members, neglecting the general subject of medicine, dazzled by the apparent brilliancy of $z$ select few, will wander forth to ultimately destroy their own usefulness. The American Medical Association is the representative body of the medical profession of this country, and has been organized and conducted by gentlemen who have grown gray in its service, and whose brows are adorned with chaplets most worthily won, not only at home but abroad. It is for them, and for ynu, my fellow-members, to control the means of disseminating our science, and not for a few, who, at best, only form but a spoke in the wheel of the triumphant chariot of our science. Our young men should learn from you that knowledge must come to them by the coopperation of their professional brethren from all parts of our country rather than from a few, who, in their exclusiveness, set themselves above the active members of the greatest and most humanitarian of all professions.

\section{MEDICAL PROGRESS.}

\section{ANATOMY AND PHYSIOLOGY,}

Anatomical Injection of the Blood-Vessels Through the Cerebro-Spinal Cavity.-Dr. $B$. W. Richardson, in his Asclepiad, gives the curious results of some experiments made by him several years ago. He was trying to stimulate the brain directly with oxygen gas, and used for the purpose a long subcutaneous injection needle introduced into the cavity of the cranium from behind the eye ball, through the optic foramen. In his operations on animals, on opening the abdomen to expose the iliac veins and inferior cava, they were found to be filled with dark blood, then, on using his injection, almost instantly the blood in the vena cava inferior became of bright red arterial color, the color gradually extending along the vessel, until, in time, it reached the right side of the heart. This effect was produced by the use of oxygen, of common air, and of carbonic oxyde. The venous blood in the senuses of the brain and in the vena cava superior was not changed in color by the injection. Next, after dividing the vena cava and inserting a tube, coal-gas was injected in the same way, and escaped so freely from the vena cava jet that it could be lighted immediately. A drachm of mercury was made to pass in the same way into the iliac veins and lower part of the vena cava, this was repeated until the mercury reached the heart, traversed the lungs through the pulmonary circuit, reached the left side of the heart and extended into the arteries. In the process of embalming, four pints of the preserving fluid can be injected over the body through the subcutaneous injecting needle, by the cerebro-spinal cavity.

Dr. Richardson has been unable to discover, up to this time, how, from the cerebro-spinal cavity, the injected matter enter the veins so easily. It charges the plexuses of the medulii-spinal veins which lie between the pia mater and the arachnoid, but the entrance of it is obscure. No injury is traceable in the brain substance after the injection, unless undue force is used, but it is obvious that the lateral ventricles are first charged with the injection.

This leads to the almost certain influence that the cerebro-spinal fluid, which is always being secreted, finds its return current into the venous circulation in a similar manner, and that the cord and brain are thus relieved of pressure from it. If that be so, we have a ready and rational explanation of many cerebral and spinal disturbances occurring under retarded current of blood through the vena cava inferior by pressure in the vessel, or by obstruction in it in some part of its course. He gives as a case in point that of a gentleman advanced in life who was laboring under ascites. The abdomen was tense with the fluid, but the urgent and apparently fatal symptoms were cerebral and spinal. The patient was quite unconscious, breathed with deep stertor, and had frequent convulsive starts and tremors. Being in consultation, he suggested that the nervous symptommight be due to obstructed venous circulation and what might be called cerebro-spinal dropsy, and advised tapping, which was done with the result of drawing off eight pints of fluid, and restoring the patient to consciousness. He lived for nearly two years afterwards in comparative comfort, undergoing the operation of tapping several times, and sinking at last from gradual exhaustion increased by senile degeneration. Dr. Richardson thinks it probable that the vertigo which is met with in temporary hepatic obstruction, is often due to retardation of the return ascending venous current, and suppression of the cerebro-spinal secretion; as also hysterical convulsion, preceded by great distension of the abdomen from flatus, and that puerperal convulsion may be mechanically induced in the same manner, as the discharge of the cerebro-spinal fluid into the general circulation is seen to be, chiefly, at a part of the venous system where uterine pressure is most likely to prove an impediment to the ascending venous current. 